East African Medical Journal Vol. 77 No. 4 April 2000

LAPAROSCOPIC CHOLECYSTECTOMY AT THE AGA KHAN HOSPITAL, NAIROBI.

S. C. Patel, FRCS, FICS, Consultant Surgeon and Chairman, Department of Surgery and J. R. Bhatt, MBChB, Senior House Officer, Department of Surgery, The Aga

Khan Hospital, P.O. Box 46356, Nairobi.

\title{
LAPAROSCOPIC CHOLECYSTECTOMY AT THE AGA KHAN HOSPITAL, NAIROBI
}

\author{
S. C. PATEL and J. R. BHATT
}

\begin{abstract}
Objective: To evaluate our experience of laparoscopic cholecystectomies at the Aga Khan Hospital, Nairobi over a three-year period from the inception of the technique, and to assess its value and advantages to the patients.

Design: A prospective case series study.

Setting: The Aga Khan Hospital, Nairobi.

Patients: One hundred and thirty five cases operated from February 1996 to April 1999. All patients were subjected to the American method of laparoscopic cholecystectomy, which is described in detail in this paper.

Main outcome measures: Clinical presentation, age and sex demographics, average hospital stay, intraoperative and postoperative complications and outcome.

Results: There was a female preponderance with a female to male ratio of 5:1. Mean age was forty nine years. Majority of patients suffered from chronic cholecystitis. The conversion rate to an open procedure was five per cent. There were two cases of significant bile leakage which required laparotomy. No mortality was reported in this series.

Conclusion: This technique was found to have distinct advantages such as shorter hospital stay, lesser postoperative pain and very good cosmesis. It is a safe procedure if performed by a well trained surgeon.
\end{abstract}

\section{INTRODUCTION}

Gall stones date back to antiquity, and were observed in the mummified corpses of the Egyptian dynasty(1). In 1882, Carl Langenbuch performed the first open cholecystectomy in Berlin. He did more than remove the first gallbladder - he enunciated a principle that: "the gallbladder needs to be removed not because it contains the stones, but because it forms them"(2). Over the years, open cholecystectomy has become the gold standard in management of gallstone disease.

Nothing over the last three decades has gripped the interest and fired the imagination of the surgical community as greatly as the laparoscopic technique. The first laparoscopic cholecystectomy was performed by Dr Phillip Mouret in Lyons, France in March 1987. By 1989, the first report of 63 cases by Perissat in France came out(3). Soon, various centres in Europe and North America started training in laparoscopic cholecystectomy. To date, the popularity of this technique has grown exponentially. It has become the routine standard method in the western world. It is however slowly being introduced in the third world countries, with South Africa and Zimbabwe(4) being the first African countries to adopt this new technique.

Widespread experience indicates that this operation when performed by a well trained surgeon is safe. Currently there are no absolute contraindications for laparoscopic cholecystectomy, but there are some relative ones, the determination of which depends on the operator's experience. These include generalised peritonitis, septic shock from cholangitis, shock from severe acute pancreatitis, cirrhosis with portal hypertension, severe coagulopathies, cancer of gallbladder and third trimester pregnancy. This technique carries several and significant advantages in terms of less postoperative pain, shorter stay in hospital, absence of wound complications, better cosmetic effect, earlier recovery and return to work.

In a developing country, every small step forward is faced with barriers and economic problems of unimaginable magnitude and the winds of change reach late and blow very gently. Our experience, difficulties, problems and joys as we progressed along the path of laparoscopic cholecystectomy are recorded here.

\section{MATERIALS AND METHODS}

After initial proper training, a unit to perform this procedure was set up at the Aga Khan Hospital, Nairobi. Under the guidance, training and assistance of an experienced laparoscopic surgeon from Canada, an initial 20 laparoscopic cholecystectomies were carried out. The technique and instruments used were the same in each case. All the cases were performed within one unit in the hospital and as more experience and confidence developed, the registrar and senior house officer working in our unit were allowed to do this procedure under supervision and guidance. Initial training of theatre support staff was not easy, but after training and experience in handling the instruments, the present staff is doing an expert job.

Since the hospital has invested in newer laparoscopic hardware comprising $30^{\circ}$ scope, three microchip camera, xenon 
light source, electronic gas chamber and $50 \mathrm{~cm}$ monitor, our innumerable problems with previous hardware have disappeared and now the system is functioning smoothly and efficiently.

The majority of our patients presented with epigastric or right upper quadrant pain (biliary colic). Other symptoms included nausea, vomiting, anorexia, fever and shivering. Cases with obstructive jaundice were not included in this group. A base line haemogram, urea, electrolytes, creatinine, fasting blood sugar and liver function tests were done in all cases. Ultrasonography of liver, gallbladder and common bile duct was done in all cases and was diagnostic of gall stones, inflammation or distension of the gallbladder. ERCP was done preoperatively in cases of stones in the common bile duct. This is also a newly introduced procedure at the Aga Khan Hospital since June 1998. Gross obesity was no contraindication.

We followed the American technique(5). Patients were placed in supine position and subject to general anaesthesia. The position of the patient in relation to the staff and equipment is shown in Figure 1. In all cases, a nasogastric tube was inserted just before surgery to decompress the stomach from the field, and removed at the end of the operation. In order to avoid possible complications of blind insertion of Veress needle and the first trocar, such as perforation to aorta, vena cava, mesenteric vessels or bowel, that is, injury to aorta, vena cava and other retroperitoneal vessels; injury to mesenteric vessels; bowel injuries and; bladder perforation, we perform open pneumoperitoneum, using the Hasson technique(5).

\section{Figure 1}

Position of patient in relation to surgeon $(S)$, assistants (A1) (A2), anaesthetist (AN), scrub nurse $(N)$, and monitor $(M)$. Ideally there should be two monitors on either side. Also note positions of the four ports on the abdomen.

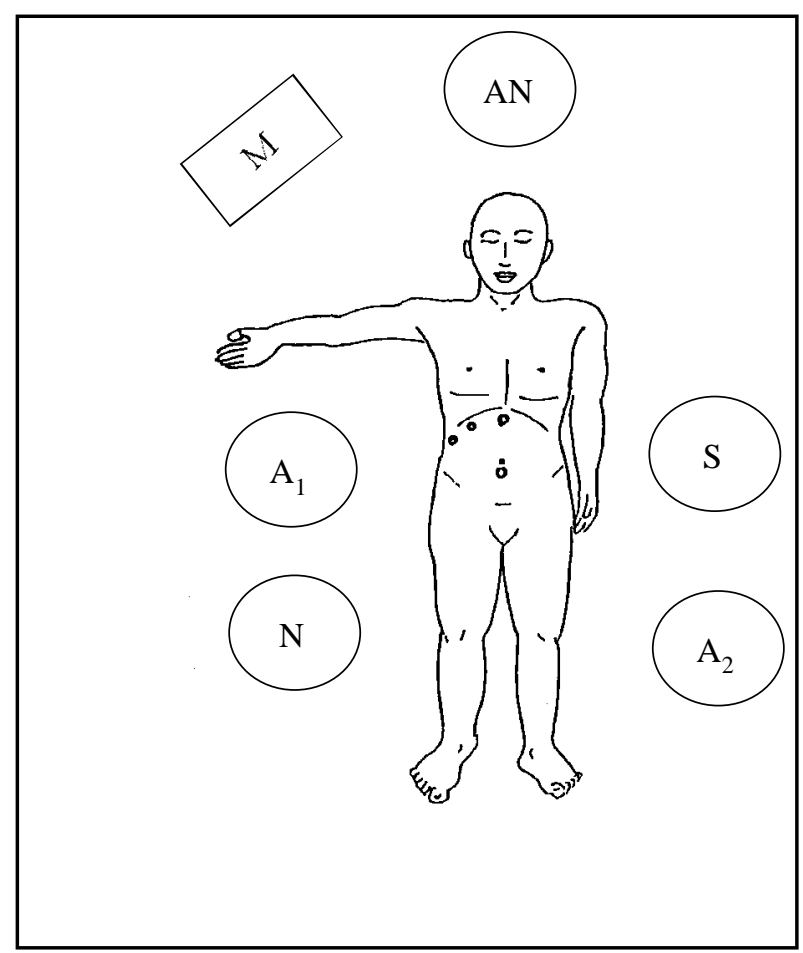

Here, a small incision of about $1 \mathrm{~cm}$ is made in the midline just above or below the umbilicus and rectus sheath and the peritoneum is opened under direct vision. Hasson's trocar which is $10 \mathrm{~mm}$. in size, is introduced and the abdomen is then insufflated with carbon dioxide to a maximum pressure of $15 \mathrm{~mm}$. $\mathrm{Hg}$. and a total of 3 litres of gas is filled in.

The $30^{\circ}$ laparoscope is then introduced through Hasson's cannula and the abdominal cavity is inspected and feasibility of the procedure determined. All the remaining trocars are inserted under direct vision. A second $10 \mathrm{~mm}$ working trocar is introduced below the xiphisternum and to the right of the falciform ligament and at the lower border of the liver. Two $5 \mathrm{~mm}$. trocars are introduced at the subcostal level, one in the mid clavicular line and the other at the anterior axillary line. A grasper is then introduced through the last trocar and the fundus of gall bladder is grasped and pushed upwards and laterally towards the right shoulder, hence retracting the liver as well. The second grasper is introduced through the mid clavicular port and grasps the Hartman's pouch, pulling it inferolaterally and thus opening up Calot's triangle.

Dissection of the hilum should start at the infundibulum of the gall bladder cystic duct junction. We use a curved dissector or a hook. Overlying fat should be grasped and pulled downwards until cystic duct is seen. Meticulous dissection close to the junction of the cystic duct and gall bladder is safer than dissection nearer the common bile duct. Once the cystic duct is clearly identified dissection of cystic artery is performed again closer to the gall bladder. Three titanium clips are placed on both cystic duct and artery and each structure is divided with curved pair of rotating scissors, leaving two clips on the proximal end (Figure 2).

\section{Figure 2}

(A) dissection of calots triangle and clipping of cystic duct and artery; $(B)$ dividing cystic duct and artery after clipping; $(C)$ dissection of gall bladder out of liver bed with hook and diathermy
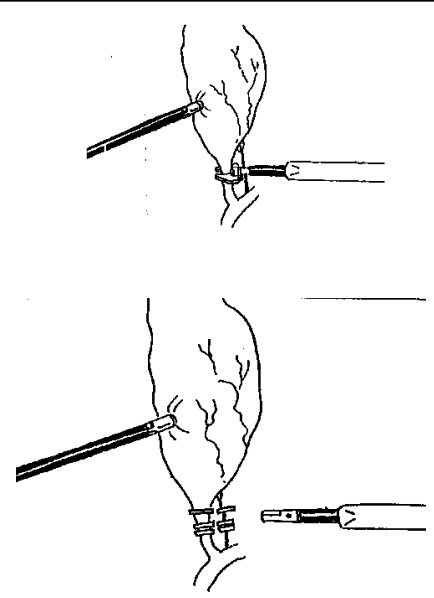

(B)

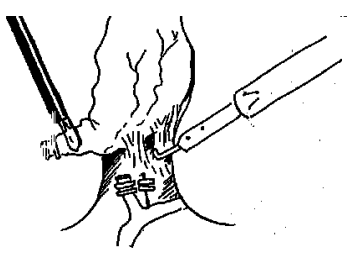

(C)

We inject $20 \mathrm{cc}$ of normal saline at the subserosal level of the gallbladder and then dissect it out from the liver bed with a 
hook diathermy. All bleeders are coagulated and gallbladder fossa irrigated with saline. The gallbladder is then removed through the umbilical port using 'crocodile' forceps after which all ports are withdrawn and the wounds are closed.

In this series we did not perform any operative cholangiogram. We give prophylactic antibiotics of three doses of cephalosporins in the intra- and post operative periods in all the routine cases. Acute cholecystitis and empyema are treated with a full course of antibiotics for at least one week.

\section{RESULTS}

There were 135 cases from February 1996 to April 1999, with an average of three to four cases a month. Out of these, 107 were female patients amounting to $79 \%$, while 28 or $21 \%$ were males, hence a female to male ratio of 5:1 (Figure 3). The age range was from 14 years to 70 years, with a mean age of 49 years (Figure 4 ).

\section{Figure 3}

Sex distribution
There were 112 cases of chronic cholecystitis, of which 80 presented as right upper quadrant pain, while 32 as biliary colic. Acute cholecystitis accounted for 19 cases while there were only two cases each of empyema of gallbladder and acalculous cholecystitis (Table 1).

\section{Table 1}

Indications

\section{Chronic cholecystitis}

(a) RUQ pain

(b) Biliary colic

Acute cholecystitis

Empyema

Acalculous cholecystitis

Operative time was initially three hours but now takes an average of one hour having acquired more experience. We had to convert seven cases to open again mainly in the initial stages. This amounts to five per cent conversion rate. Reasons for conversion included severe adhesions in four cases, unclear anatomy and poor visibility in two cases and cystic artery rupture in one case (Table 2).

Table 2

Reasons for conversion to open

\begin{tabular}{ll}
\hline Severe adhesions & 4 \\
Unclear anatomy/poor visibility & 2 \\
Cystic artery rupture & 1
\end{tabular}

Figure 5

Possible reported common bile duct and hepatic duct injuries. Note that we did not have any such injuries in this series

(a) common hepatic duct; (b) common bile duct; (c) cystic duct junction

\section{Figure 4}

Age distribution

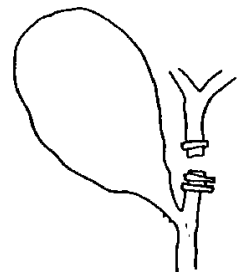

(A)
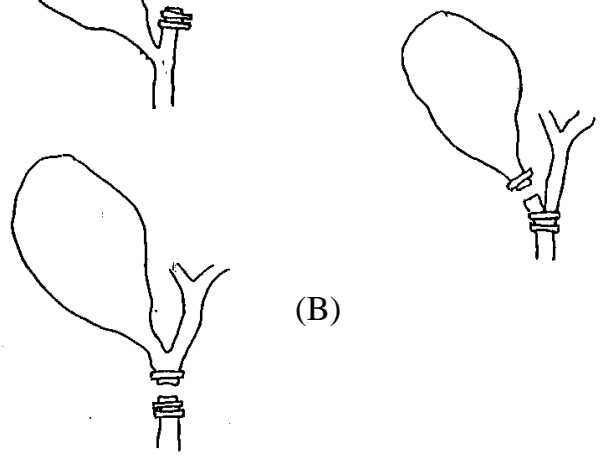

(C)

(B)

Average stay in hospital was two days with the vast majority of patients returning to their normal activities and work within a week. No mortality was recorded in this 
series in the first 30 days post operatively. There were two cases of pneumonia (one case was HIV positive) and two cases of umbilical wound infection. All these recovered with antibiotics.

There was one case of a minor bile leak which resolved spontaneously. Major bile leak leading to biloma was found in two cases one week after surgery, both of which required a laparotomy. This was due to diathermy injury to common bile duct at the cystic duct junction. After inserting a T- tube through the sloughed area of common bile duct it healed well and both patients recovered completely. There were no cases of common bile duct or right hepatic duct transection or clipping in our series.

The gall bladder was perforated in 17 cases during removal from liver bed, resulting in bile spillage. In each case large stones were picked where present, subhepatic space was irrigated with saline wash and the patient was covered with cephalosporin antibiotics for a week.

Post operatively, 18 cases developed mild pain in right upper quadrant which resolved spontaneously within a week. Two cases were readmitted with biliary colic, for which ERCP was done in one. This did not show any stone in CBD and patient's symptoms subsequently resolved. The other case improved on omeprazole for associated duodenal ulcer.

\section{DISCUSSION}

Open cholecystectomy has been a gold standard for symptomatic gall stones since $1882(1,2,6)$. However in the last ten years, laparoscopic cholecystectomy has superceded open surgery in the western world. The widespread acceptance of this method by both surgeons and patients alike is one of the most unparalleled phenomenon in the history of surgery. Even in the developing countries, as more people become aware of the availability of such a procedure, the demand of laparoscopic surgery by patients is increasing day by day.

In our series, the indications ranged from chronic cholecystitis and biliary colic (majority), acute cholecystitis, empyema of gall bladder and acalculous cholecystitis. Currently there are no absolute contraindications, but there are some relative ones depending on the operator's experience.

We have used the American technique of laparoscopic cholecystectomy(5). This is a relatively safe procedure in experienced hands but requires proper training. Our present operative time averages one hour. We have adopted the open technique of Hasson(5) which we feel is safer than the Veress needle insertion. The Hasson technique avoids blind insertion of the first port and hence complications related to needles and trocars are avoided $(7,8)$.These include injuries to major vessels, bowel, bladder and the mesentery. Vascular injury usually occurring during placement of the first trocar has a mortality of $9 \%$ and is the most common lethal complication of laparoscopic cholecystectomy $(9,10)$. Bowel injuries associated with a $5 \%$ mortality rate can occur during insertion of needles and trocars, as well as by improper use of diathermy and laser(9). Localised tissue damage is less likely to occur with diathermy(11), which has been proved to be the most rapid and haemostatic method of dissection $(11,12)$. No such complications were seen in this series.

Conversion to open cholecystectomy in our series amounted to 5\%, which is comparable with large series internationally with reported conversion rates of between 4 and $7 \%(13,14)$. Conversion to open procedure is usually seen more in the beginning of the learning curve, and is noted to reduce once the operator gains experience and confidence. In this series, there were no conversions in the last 50 cases performed. Reasons for conversion to open are listed in Table 2.

There was no mortality reported in this series. Mortality has decreased since the first cholecystectomy in 1882 . Currently it is regarded as a safe procedure with an average mortality of $0-1.8 \%(9)$. The risk factor that consistently correlates with operative mortality is the patient's age(9); the risk being 17 times greater in patients aged over 65 years $(0.5 \%)$ compared with younger patients $(0.003 \%)(15)$.

Morbidity occurs generally in $5-15 \%$ of patients with the open technique $(15,16)$. This is frequently due to wound infection, sepsis, common bile duct injuries, retained stones and associated medical complications, such as chest infection, cardiovascular accidents and urinary problems. With the laparoscopic technique, morbidity is mostly related to technical complications(16), which can be broadly divided into insertion of needles and trocars already discussed earlier, the creation of pneumoperitoneum or the improper use of equipment during dissection of the cystic duct resulting in bile duct injuries.

The creation of pneumoperitoneum during carbondioxide insufflation can cause complications, adding to the usual anaesthetic hazards a risk of cardiac arrhythmias or arrest, especially if the intra-abdominal pressure rises excessively $(17,18,19)$. This is avoided by using low pressure pneumoperitoneum (12 $\mathrm{mmHg}$ or less) and by very strict observations and monitoring during laparoscopic anaesthesia(19).

Injury to the bile duct during the procedure is an inherent complication(20,21). The first case of bile duct injury was recorded by Mayo in 1905(22). Reported cases of common bile duct injuries during laparoscopic cholecystectomy have ranged from $0.2 \%$ to $3.4 \%$, this being much higher than the $0.1 \%$ to $0.2 \%$ encountered during open cholecystectomy(16,22-26). The rate of bile duct injuries appears to be decreasing as experience is gained with this new technique. Biliary leakage which often occurs following bile duct injury is present in 0.3$0.6 \%$ of patients $(27,28)$. When this is free leakage, it can cause bile ascites, biliary peritonitis or external fistula. If the collection is localised, it produces a biloma.

We encountered one minor bile leak postoperatively which resolved spontaneously, while there were two major leaks with biloma formation both needing laparotomy. These were due to diathermy injury to the common bile duct at the cystic duct junction. At present, we do not use diathermy near the Calot's triangle and the whole dissection of the cystic duct and artery is carried out with a Maryland dissector, thus avoiding the possibility of thermal injury to 
vital structures. We also follow Hunter's principle(12) and stay closer to the junction of the infundibulum of the gall bladder and cystic duct during dissection and clipping, thus avoiding possible common bile duct damage. Possible reported bile duct and hepatic duct injuries are shown in Figure 5. Note that there were no cases of bile duct or hepatic duct transection or clipping in our series.

There was no case seen with postoperative retained stone in the common bile duct in our series. Studies have shown that in open cholecystectomy and bile duct exploration, stones will be retained in seven to ten per cent of patients $(16,29,30,31)$. Careful preoperative workup of patients was done in this series using investigations such as ultrasonic measurement of common bile duct, liver function tests and a baseline haemogram. Any patients with features of obstructive jaundice or common bile duct dilatation of more than $8 \mathrm{~mm}$ on ultrasound was subjected to a preoperative ERCP and removal of stones, failure of which would then necessitate open surgery.

In open cholecystectomy, most complications are related to the laparotomy scar such as wound sepsis, dehiscence and late incisional hernia(32). Abdominal surgery also leads to restrictive impairment of pulmonary function(33,34). Comparative studies of laparoscopic and open cholecystectomies have shown better postoperative pulmonary function using the laparoscopic approach(35).

Laparoscopic cholecystectomy has many distinct advantages. Postoperative pain is much less, especially since the incisions are no more than $10 \mathrm{~mm}$, whereas the Kocher's incision entails splitting or dividing the abdominal muscles, hence contributing to pain. There are lesser chances of wound sepsis and no dehiscence with the laparoscopic method. Hospital stay is reduced as well. In our series, the average hospital stay was two days. With open cholecystectomy, the patient usually stays in for 5-7 days, hence increasing the financial burden, notwithstanding the psychological impact that prolonged hospital has on the patient. Laparoscopic cholecystectomy allows earlier return to normal activities and work. Aesthetically, there is very minimal scarring and excellent cosmesis as well.

In conclusion, we consider that the benefits of laparoscopic surgery are even greater in the developing world, where demand for hospital beds is great, health insurance cover almost non-existent for the common man, and early return to work a financial necessity. With this in mind, we strongly recommend that this novel technique be given more importance in the training of surgeons in this part of the world.

\section{ACKNOWLEDGEMENTS}

To the Medical Director, the Aga Khan Hospital, Nairobi for permission to publish this study.

\section{REFERENCES}

1. Udwadia T.E. Laparoscopic Cholecystectomy, 1991.

2. C. Langenbuch. A case of gall bladder removal for chronic cholelithiasis: Klinishe Wochenshrift. 1882; 19:725.

3. Perissat, J. Collet, D. and Belliard R. Gallstones: Laparoscopic Treatment cholecystectomy, cholecystostomy and lithotripsy.-Our own technique. Surg. Endosc. 1989; 3:131.
4. Mauchaza et al. Laparoscopic cholecystectomy in Zimbabwe. East Cent. Afr. J. Surg. 1995; vol:2.

5. Phillip and Rosenthal.- Operative strategies in laparoscopic surgery, 1995.

6. Munson J. and Sanders L. Open cholecystectomy revisited. Surg. Clin. N. Amer. 1994; 74:741-754.

7. Isla A. and Thomas K. et al. Potential complications of cholecystectomies. Intern. J. Gastroent. 1998; 9-11.

8. Geers J. and Holden C. Major vascular injury as a complication of laparoscopic surgery. A report of 3 cases and review of the literature. Amer. J. Surg. 1996; 62:377-379.

9. Deziel D.J. Complications of cholecystectomy incidence, clinical manifestations and diagnosis. Surg. Clin. N. Amer. 1994; 74:809-823.

10. Deziel D.J. and Millikan K.W. et al - Complications of laparoscopic cholecystecomy: a national survey of 4,292 hospitals and ananlysis of 77,604 cases. Amer. J. Surg. 1993; 165:9-14.

11. Bismuth H. and Franco D. et al - Long term results of Roux-en Y hepaticojejunostomy. Surg. Gynec. Obstet. 1978; 146:161-167.

12. Hunter J.G. Exposure dissection, and laser versus electrosurgery in laparoscopic cholecystectomy. Amer. J.Surg. 1993; 165: 492-496.

13. Chime and Studley. Audit of methods of laparoscopic cholecystectomy. Brit. J. Surg. 1999; 86:185-188.

14. Geoghegan J.G. and Keane. Laparoscopic management of complicated gall stone disease. Brit. J. Surg. 1999; 86:146-148.

15. Roslyn J.J., Binns et al - Open cholecystectomy. A contemporary analysis of 42,474 patients. Ann. Surg. 1993; 218:129-137.

16. Habib N.A. and Foo C.L. et al. Complications of cholecystectomy in district general hospitals. Brit. J. Clin. Pract. 1990; 44:189-195.

17. Mirghani O.A. and Babiker M.Y. Experience with gynaecological laparoscopies in Wad Medani Hospital, Sudan. East Afr. Med. J. 1999; 76: 390-395.

18. Harris M.N.E., Planteven et al - Cardiac arrhytmias during anaesthesia for laparoscopy. Brit. J. Anaesth. 1984; 56:1213-1217.

19. Lenz R.J. and Thomas T.A. et al - Anaesthesia for laparoscopy. Anaesthesia. 1970; 31:4.

20. Bismuth H. Postoperative strictures of the bile duct- Surgery of the liver and biliary tract, Churchill Livingstone 1982;5:209-218.

21. Strasberg S.M., Hertl m. and Soper N.J. An analysis of the problem of biliary injury during laparoscopic cholecystectomy. J. Amer. Coll. Surg. 1995; 180:101-125.

22. Mayo W.J. Some remarks on cases involving operative loss of continuity of the common bile duct with a report of case of anastomosis between the hepatic duct and the duodenum. Ann. Surg. 1905; 42:90-96

23. Peters J.H. and Gibbons G.D. et al Complications of laparoscopic cholecystectomy. Surgery. 1991; 110: 769-780.

24. Csendes A. and Diaz J.C. et al - Late results of primary repair and follow-up in 53 patients with injuries to the common bile duct occuring during cholecystectomy. Hepatogastroenterology. 1994; 41: $195-200$.

25. Raute M. and Podlech P. et al - Management of bile duct injuries after laparoscopic cholecystectomy. Wld. J. Surg. 1993;17: 553-562.

26. Schol F.P.G. and Go P. et al. Outcome of 49 repairs of bile duct injuries after laparoscopic cholecystectomy. Wld J. Surg. 1995; 19:753-757.

27. Clavien P.A. and Sanabria J.R. et al - Recent results of elective open cholecystectomy in a North American and a European Center. Ann. Surg. 1992; 216: 618-626.

28. Crist D.W. and Gadazc T. R. Complications of laparoscopic surgery. Surg. Clin. N. America. 1993; 73: 265-289.

29. Schwartz D.I. Gallbladder and extrahepatic biliary system. Principles of Surgery, 4th edition, McGraw Hill -1307-1343 (1974).

30. Longmire W.P. The diverse causes of biliary obstruction and their remedies. Curr. Probl. Surg. 1977; 14: 1-59.

31. Glenn F. Postcholecystectomy choledocholithiasis. Surg. Gynecol. Obstet. 1972; 176: 374-351.

32. Litwin D.E.M., Girotti et al - Laparoscopic cholecystectomy: TransCanada experience with 2201 cases. Canadian J. Surg. 1992; 35:291-296.

33. Latimer R.G. and Dickman M. et al - Ventilatory patterns and pulmonary complications after upper abdominal surgery determined by pre- and postoperative computerized spirometry and blood gas analysis. Amer. J. Surg. 1971; 122:622-632.

34. Craig D.B. Postoperative recovery of pulmonary function. Anaesth Analg. 1993; 60: 46-52

35. Coelho J.C.U. and de Araujo R.P.M. et al - Pulmonary function after cholecystectomy performed through Kocher's incision, a mini incision and laparoscopy. Wld J. surg. 1993; 17: 544-546. 